\title{
ADAPTIVE PROBABILISTIC NAV TO INCREASE FAIRNESS IN AD HOC 802.11 MAC LAYER
}

\author{
Claude Chaudet ${ }^{1}$, Guillaume Chelius ${ }^{2}$, Hervé Meunier ${ }^{3}$, David Simplot-Ryl ${ }^{3}$ \\ ${ }^{1}$ GET-ENST ; LTCI-UMR 5141 CNRS, 46, rue Barrault, 75634 Paris, FRANCE- \\ Claude. Chaudet@enst . fr \\ ${ }^{2}$ CITI/INRIA ARES - INSA de Lyon-Bat L. de Vinci-21, avenue J. Capelle - 69621 Villeur- \\ banne, FRANCE - guillaume , chelius@inria.fr \\ ${ }^{3}$ IRCICA/LIFL, Univ. Lille 1, CNRS UMR 8022 - INRIA Futurs, POPS research group - Bat. \\ M3, Cite Scientifique - 59655 Villeneuve d'Ascq Cedex, FRANCE - \\ \{meunier, simplot\}@lifl.fr
}

\begin{abstract}
The IEEE 802.11 MAC layer is known for its low performances in wireless ad hoc networks. For instance, it has been shown in the literature that two independent emitters nodes can easily monopolize the medium, preventing other nodes to send packets. The protocol we introduce in this article is a simple variation of the original IEEE 802.11 MAC layer which significantly increases the fairness while maintaining a high effective bandwidth. Its principle consists in avoiding systematic successive transmissions by the same emitter through the probabilistic introduction of a waiting time, a virtual NAV, after each emission. The probability to set a NAV is adaptively computed depending on the perceived utility of the previous virtual NAV. This protocol, called PNAV (Probabilistic $N A V$ ), is shown to be efficient by simulation and is compared to another IEEE 802.11 adaptation.
\end{abstract}

\section{Introduction}

Medium-access control (MAC) protocols for wireless networks have received a considerable attention over the past few years with the aim to reduce the number of collisions while maximizing the bandwidth use. Collisions occur when a node is in the neighborhood of two simultaneous transmitters. If the transmitting stations are neighboring nodes, the collision probability can be reduced through the use of a simple random backoff algorithm and a carrier sense mechanism. These principles are the basics of the widely used CSMA protocol family. If the transmitting stations cannot communicate directly, the 
collision risk is increased due to the absence of carrier sense. This problem was first described by [Kleinrock and Tobagi, 1975], and is known as the "hidden terminal problem". Several solutions have been proposed to resolve this problem. For instance, communicating nodes can exchange short control messages to inform their neighborhood of the forthcoming data frame. In the IEEE 802.11 Distributed Coordination Function (DCF), a node initiating a communication first sends a request-to-send (RTS) frame to the receiver. If the intended receiver correctly receives the RTS frame and if the medium is free in its vicinity, this receiver answers with a clear-to-send (CTS). Upon reception of the CTS frame, the sender transmits its data frame. The RTS and CTS control frames contain the duration of the subsequent data exchange, which gives the opportunity to all neighboring nodes to be aware of the medium occupation induced by the communication. More precisely, nodes that receive RTS and/or CTS frames set a "Network Allocation Vector" (NAV) for the duration of the exchange and will restrain from transmitting during this period.

In addition to collisions, the hidden terminal situation is responsible for several issues. [ $\mathrm{Ng}$ and Liew, 2004] have shown that along a node string in a multihop network, all nodes do not have the same medium access. The unfairness of the MAC protocol is also clearly exhibited by [Chaudet et al., 2005b]. They propose a simple scenario with three pairs of emitters and receivers where two pairs capture the totality of the medium while the third one has no opportunity to compete for the medium access. Such typical scenarios appear when the medium is saturated. There have been some proposals to solve these issues and they usually lead to a traffic limitation. In this paper, we address the fairness problem while ensuring an efficient use of the channel bandwidth. We propose a simple modification of the MAC layer where nodes can probabilistically set a virtual NAV after each sent frame. The probability to introduce such a NAV is adaptively computed according to its observed utility. We show that our approach is efficient compared to already existing solutions. This MAC protocol is called PNAV for Probabilistic NAV and is fully compatible with the IEEE 802.11 standard.

A literature study and a full description of IEEE 802.11 can be found in the extended version of this article, [Chaudet et al., 2005a]. The remainder of the paper is organized as follows: We describe our proposal in Section 2. In Section 3, we describe the simulation environment and results are given in Section 4. Section 5 concludes the paper and presents the future works.

\section{Probabilistic NAV}

Under certain circumstances, the IEEE 802.11 DCF function leads to an unbalanced bandwidth repartition or different medium access probabilities between different radio links. For example, let us consider the three pairs topol- 
ogy intensively studied in [Chaudet et al., 2005c, Chaudet et al., 2005b] and showed on Figure 1. In this scenario, three emitters contend for medium access. The topology is unbalanced and one emitter competes with the two others while the other ones only have to deal with the central emitter. Neighbor emitters are in mutual carrier-sense range but cannot directly communicate.

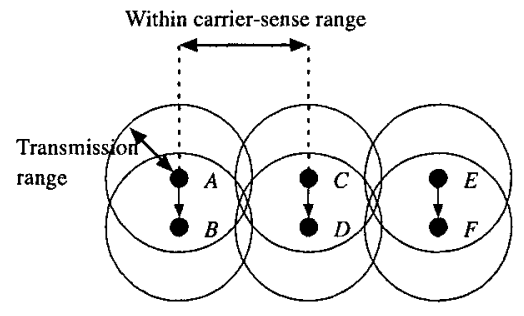

Figure 1. 3-pair topology.

The pair in the middle almost never gains access to the medium that is monopolized by the exterior pairs. In this configuration, the exterior pairs do not even get knowledge of the middle one trying to access the medium. In consequence, IEEE 802.11 DCF adaptations such as AOB ([Bononi et al., 2004]) that estimates the medium occupation using emitters' perception of the state of the medium, only slightly increase the fairness of IEEE 802.11 in this situation. As the middle pair is dumb, exterior pairs do not hear any other communications, do not delay transmission through the classical backoff or NAV mechanisms and thus consider the medium unoccupied which in turn reduce the efficiency of other enhancement proposals.

In the presence of dumb radio links, the only way to increase the fairness is to give these links an opportunity to express themselves. This may be done through the introduction of a NAV in the IEEE 802.11 layer of nodes that frequently access the medium. These silence periods may give the opportunity to dumb radio links to transmit packets and to notify their presence to all surrounding nodes that could in turn activate mechanisms to increase fairness.

Our proposal follows this strategy. According to a varying probability, a node sets a NAV of duration $\delta$ after each transmission in order to give other nodes the possibility to gain access to the medium. The NAV probability, $p_{n a v}$, is a function of both the node and other nodes' use of the medium. Qualitatively, it helps emitters answer questions such as "am I monopolizing the medium?" or "did my last NAV give an opportunity to another node's communication?".

In order to estimate the medium occupancy induced by a particular node, we identify three different events. These events will be used by the PNAV 
automaton to adapt the node probability to introduce a NAV after each of its emissions. Initially, the NAV probability, $p_{\text {nav }}$ is set to 0 , meaning that no NAV is introduced before the first emission.

- $t$ event: the $t$ event occurs when the considered emitter acquires the medium for two successive transmissions with an inter-emission period inferior to the NAV duration, $\delta$. If a node $u$ only keeps on experiencing $t$ events, it means that it is monopolizing the medium, and it should therefore increase its probability to release the medium. Upon observation such an event, the $p_{\text {nav }}$ probability is increased by a value $p_{s t e p}$, parameter of the protocol.

- $r$ event: the $r$ event occurs under two conditions. First, the considered mobile has set a probabilistic NAV after its last emission; second, the medium has been acquired by another node before the NAV expiration. Occurrence of an $r$ event means that the introduction of the NAV has been successful in term of medium fairness, as this silence period has been used by another node to access the medium. Therefore, after an $r$ event, $p_{\text {nav }}$ is set to 1 meaning that a NAV is systematically set as long as it is useful to encourage nodes to delay their transmissions to help starved nodes to access the medium.

- $s$ event: the $s$ event occurs under two conditions. First, the considered node has introduced a probabilistic NAV after its last emission; second, it has reacquired the medium after expiration of the NAV. Occurrence of the $s$ event signifies that the introduced NAV was not necessary, as the associated silence period has not been successfully used by another node to access the medium. Therefore, $p_{\text {nav }}$ is reinitialized to 0 when an $s$ event occurs in order to ensure a low bandwidth waste if the considered node is the only one competing to access the medium.

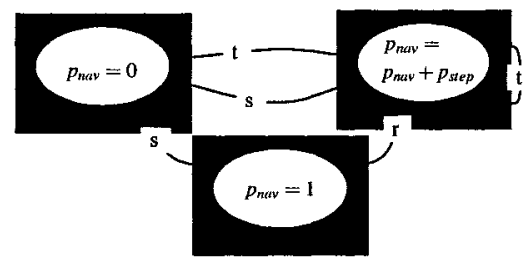

Figure 2. PNAV automaton

The automaton determining $p_{n a v}$ in function of the different events is depicted on Figure 2. The main concerns behind the automaton are to introduce 
$\mathrm{NAV}$ as soon as a node is monopolizing the medium ( $t$ event), to keep on introducing NAV if they are useful ( $r$ event) and finally not to introduce a NAV if it is useless ( $s$ event). The PNAV mechanism depends on two parameters $p_{\text {step }}$ and $\delta$.

\section{Evaluations}

This section focuses on introducing the simulation environment and the scenarios we used in order to study some equity issues observed in IEEE 802.11 DCF.

Simulations in this study involve several parallel pairs of nodes, each pair having an emitter node trying to transmit its traffic to a receiver node. For example, Figure 1 illustrates this topology considering 3 parallel pairs. We will first consider that parameters are set in such a way that an emitter node only senses and can communicate with its two closest neighbors (inter-pair distance: $150 \mathrm{~m}$, radio range: $160 \mathrm{~m}$, carrier sense: $160 \mathrm{~m}$ ). Then we will increase the carrier sense distance and finally fall to the scenarios depicted earlier. Beside the basic parallel pairs, we evaluate the impact of our proposition on chained nodes and random network topologies. These experiments are conducted as follows: given a topology of nodes (couples of emitter/receiver nodes), we basically generate a saturated traffic from the emitters to their respective receivers and we log the amount of data successfully received during the simulation process.

Simulations were performed using the network simulator NS-2 ${ }^{1}$ in version 2.27 with MAC and physical parameters tuned to reflect the HR-DSSS $11 \mathrm{Mb} / \mathrm{s}$ physical layer of IEEE $802.11 \mathrm{~b}$. This results in a transmission range of about $160 \mathrm{~m}$ and a carrier sense range of $160 \mathrm{~m}$ or $400 \mathrm{~m}$, depending on the simulated scenario. The MAC protocol presented above has been implemented into the simulator as well as the AOB flavor presented in [Bononi et al., 2004] with an ACL parameter value corresponding to the high-rate physical layer.

For the first analysis, three types of scenarios have been considered. Pairs of nodes, similar to the situation depicted on Figure 1 that will be described later, chains of flows and random scenarios. In all these scenarios, we used CBR flows saturating the medium. Due to space limitations, only the pairs scenarios results will be presented in the next sections, the other results being included in [Chaudet et al., 2005a].

The simplest scenario consists in a single emitter and a single receiver with a saturated traffic. It provides the opportunity to evaluate the maximum bandwidth provided in no-competition conditions.

The 2-pair saturated traffic scenario has also been evaluated in order to rate the maximum bandwidth over a shared channel, and thus, to rate the synchronization ability of the MAC protocol. 
The 3-pair scenario enlightens the typical issue about the fairness of most ad hoc MAC protocols. The middle emitter node has to compete for medium access, with emitters from both sides, which do not have to compete with each other. IEEE 802.11 DCF equity issues typically arise in this topology.

Further increasing the number of pairs then leads to similar fairness issues, whose characteristics depend on the number of pairs.

\section{Performance evaluation}

In this section, we present an analytical evaluation of the loss of bandwidth that can be expected on a single link and simulation results for the larger scenarios described above. Simulation results presented in this section are the average of the throughput mean and standard deviation of each flow, computed over 20 simulations, each simulation lasting 30 seconds. To evaluate the performance of the sole MAC protocol, we used a static routing agent for NS-2 developed by $\mathrm{T}$. Razafindralambo, computing offline shortest-paths between any pairs of nodes. Other sources of traffic such as ARP also have been disabled. Results presented here only concern transmissions without RTS-CTS exchange. Simulations also have been performed with RTS-CTS activated and the conclusions are similar in each of these situations, even though the overall performance is different.

\section{Single pair}

To begin with the performance analysis of the PNAV mechanism, we consider a single communicating pair. The aim of this first study is to evaluate the waste of bandwidth introduced by the probabilistic NAV when there is no contention on the medium. Indeed, PNAV decreases the maximum bandwidth that can be achieved by a single communication. Consider node $u$ communicating with node $v$ at a packet rate such that the inter-emission period is inferior to $\delta$. Node $u$ will observe consecutive $t$ transitions until it sets a NAV. This NAV will not be used by any other communication as $u$ is the only transmitting node and a $s$ transition will occur, reinitializing $p_{\text {nav }}$ to zero. The phenomenon will be repeated periodically, introducing useless NAVs, thus decreasing the effective bandwidth of the communication between $u$ and $v$. We will now try to evaluate this bandwidth waste as a function of the parameters $\delta$ and $p_{\text {step }}$.

Let $N_{n a v}$ be the random variable associated to the number of emissions between two probabilistic NAV. As there is only one pair communicating, the only possible transitions are $t$ and $r$ depending on whether or not a NAV has been introduced after the preceding emission. The automaton behavior can be modeled with a simple Markov chain described by Figure 3. In consequence, it is quite simple to compute the expected number of emissions $\mathrm{E}\left(\mathrm{N}_{\text {nav }}\right)$ between 
two NAV by solving the Chapman-Kolmogorov equations system associated to the Markov chain.

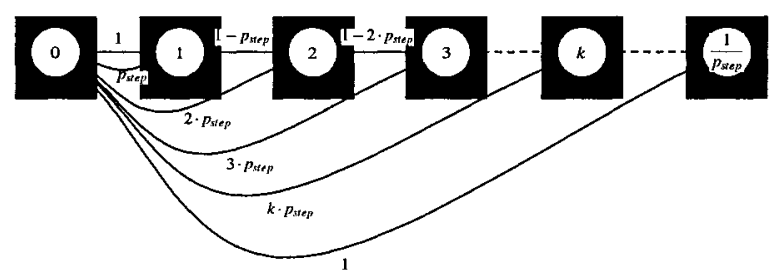

Figure 3. A markov chain describing the automaton behavior in the single pair case

$$
\begin{gathered}
p\left(N_{\text {nav }}=k\right)=p_{\text {step }} \cdot \prod_{i=0}^{k-1}\left(1-i . p_{\text {step }}\right) \\
\mathrm{E}\left(N_{\text {nav }}\right)=\sum_{k=1}^{\frac{1}{p_{\text {step }}}}\left(k \cdot p_{\text {step }} \cdot \prod_{i=0}^{k-1}\left(1-i \cdot p_{\text {step }}\right)\right)
\end{gathered}
$$

Given $\mathrm{E}\left(\mathrm{N}_{\text {nav }}\right)$ and depending on $\delta$, we can also deduce the decrease of effective bandwidth in the case of a single communicating pair. It is illustrated by figure 4. As we can see on the picture, the effective bandwidth of PNAV is close to the maximum available bandwidth $(3600 \mathrm{~kb} / \mathrm{s})$ even with large values

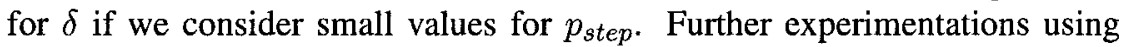
different node topologies will be carried out with $\delta=2000 \mu s$ and $p_{\text {step }}=0.1$.

\section{Multiple pairs}

We simulated two to seven parallel pairs separated by a distance close to the transmission range with a carrier sense area equal to the transmission area. Emitters only compete with their direct neighbors and no collision occurs because the receivers are near enough of their associate emitters to prevent signal jamming. This kind of scenario can happen in an indoor context, for instance. Its purpose is to give basic evaluation of the performance of the different solutions, without signal-level concerns.

Figure 5(a) presents the achieved throughput means and standard deviations as function of the number of parallel pairs. A first observation is that using PNAV leads to an almost null standard deviation, improving fairness, but at the cost of overall performance. AOB also presents a mean throughput decrease and leads to a fairness only a little better than the one achieved by IEEE 802.11.

Increasing the carrier sense range so that emitters compete for medium access with two-hops neighbors leads to the results presented on Figure 5(b). 


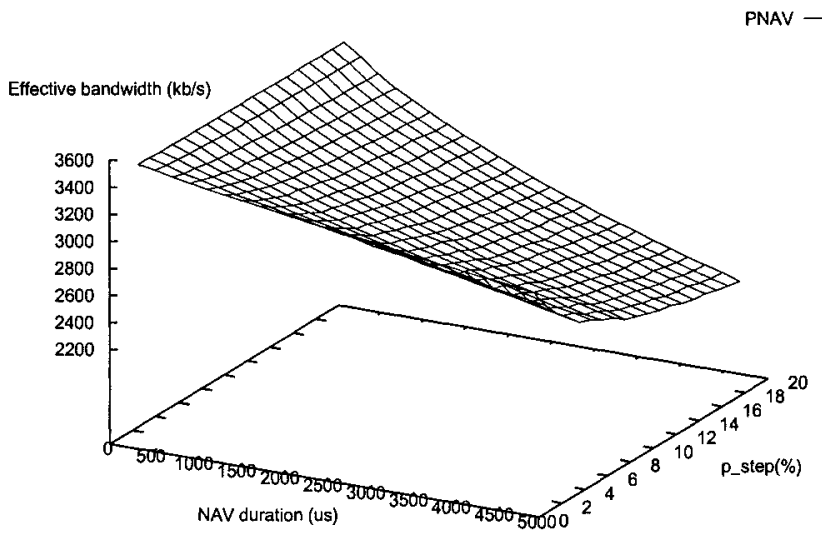

Figure 4. Effective bandwidth of a single pair using PNAV

On this figure, $\mathrm{AOB}$ and PNAV result in similar mean throughput but PNAV improves fairness. The standard deviation peaks for 4-pairs and 7-pairs configurations are due to the particularities of the topology. Let's consider, for instance the 4-pairs scenario. In this situation the central emitters have to compete with all three other emitters for medium access while the exterior ones only compete with two others. This unbalance tends to prevent central nodes from transmitting, leaving a greater share of the medium to the exterior nodes. The 7-pairs situation is indeed the aggregation of two times the 4-pairs situations. Exterior pairs and the very central pair are favored.

\section{Conclusion}

In this paper, we have presented PNAV, an adaptation of the IEEE 802.11 DCF protocol in order to increase its fairness in an ad hoc environment. Contrarily to other proposals using the medium occupation - the slot utilization metric for $A O B$ - as an input to the system, our protocol is event-driven. It consists in introducing probabilistic NAV depending on events observed on the radio medium. These events can be qualitatively described as "I am monopolizing the medium" or "my PNAV has been useful for someone's else communication". The probability to introduce a NAV evolves depending on these 


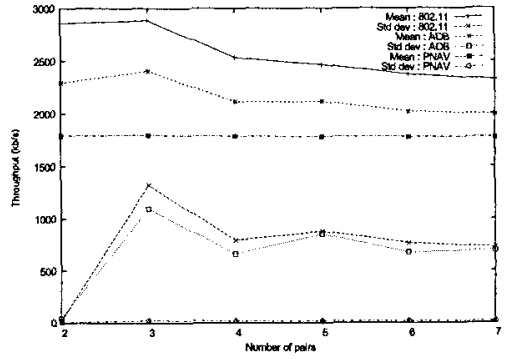

(a) $\mathrm{CR}=\mathrm{TR}$

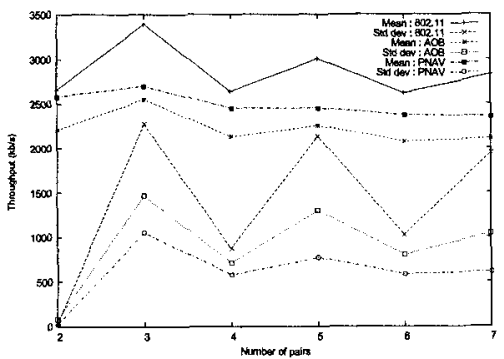

(c) no neighbor communication

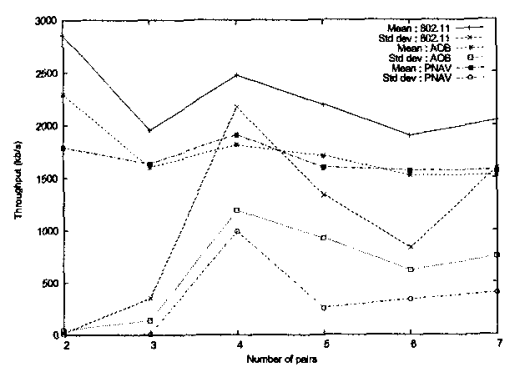

(b) Large $\mathrm{CR}$

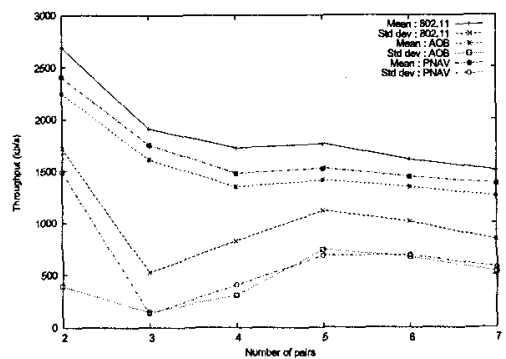

(d) 3-pairs

events. PNAV has been simulated in several topologies known for their 802.11 DCF fairness issues, the pairs and the chain topologies, as well as pseudorealistic topologies. It has shown very satisfying performances, inducing more fairness between the different flows than a classical IEEE 802.11 DCF and $\mathrm{AOB}$ as well depending on the considered topology, while maintaining a high overall throughput, lower than a classical IEEE 802.11 DCF but higher than AOB.

If the results observed by simulations are promising, several issues remain to be addressed. A theoretical analysis of the PNAV automaton and the NAV probability function is an interesting perspective as it may enlighten the existence of an optimal NAV probability as a function of the network topology, similar to the work done in [Bononi et al., 2004] in the AOB context. Other radio medium events can also be considered in order to refine the PNAV automaton with the aim to continue on increasing the MAC protocol fairness while maintaining a high achieved throughput. We also plan on studying the behavior of the proposed protocol when used in networks called heterogeneous 
by [Bruno et al., 2005], i.e. network composed of emitters using different MAC strategies.

Finally, an interesting point is that the PNAV protocol is not incompatible with other 802.11 DCF adaptations such as AOB. They present two orthogonal approaches that could be combined. While $\mathrm{AOB}$ monitors the radio occupation to adapt its deferring probability, PNAV uses different events such as successive transmissions to decide to relinquish the medium. The consequence is that both protocols show their best performances in different topologies. An interesting study would be to combine both of them in order to see whether the resulting adaptation would inherit the best of both approaches.

\section{Notes}

1. http://www.isi.edu/nsnam/ns/index.html

\section{References}

[Bononi et al., 2004] Bononi, L., Conti, M., and Gregori, E. (2004). Runtime optimization of IEEE 802.11 wireless LANs performance. IEEE Transactions on Parallel and Distributed Systems, 15(1):66-80.

[Bruno et al., 2005] Bruno, R., Conti, M., and Gregori, E. (2005). Distributed contention control in heterogeneous 802.11b WLANs. In Proc. 2nd Annual Conference on Wireless On demand Network Systems and Service (WONS 2005), pages 190-199, St Moritz, Switzerland.

[Chaudet et al., 2005a] Chaudet, C., Chelius, G., Meunier, H., and Simplot-Ryl, D. (2005a). Adaptive probability nav to increase fairness in ad hoc 802.11 mac layer. Technical report, INRIA.

[Chaudet et al., 2005b] Chaudet, C., Dhoutaut, D., and Guerin Lassous, I. (2005b). Experiments of some performance issues with IEEE 802.11b in ad hoc networks. In Proc. 2nd Annual Conference on Wireless On demand Network Systems and Service (WONS 2005), pages 158-163, St Moritz, Switzerland.

[Chaudet et al., 2005c] Chaudet, C., Dhoutaut, D., and Guerin Lassous, I. (2005c). Performance issues with IEEE 802.11 in ad hoc networking. IEEE Communication Magazine. to appear.

[Kleinrock and Tobagi, 1975] Kleinrock, L. and Tobagi, F. (1975). Packet switching in radio channels: Part $\mathbf{i}$ - carrier sense multiple-access modes and their throughput-delay characteristics. IEEE Transactions on Communications, 23(12):1400-1416.

[Ng and Liew, 2004] Ng, P. and Liew, S. (2004). Offered load control in IEEE 802.11 multihop ad-hoc networks. In Proc. IEEE International Conference on Mobile Ad hoc and Sensor Systems (MASS 2004), Fort Lauderdale, Florida, USA. 